\title{
Predictive Analytics for Disaster Management
}

\author{
${ }^{1}$ Anuja Patil \\ BE-Computer, FCRIT Vashi
}

\author{
${ }^{2}$ Kaustubh Magdum \\ BE-Computer, FCRIT Vashi
}

\author{
${ }^{3}$ Atharva Phadke \\ BE-Computer, FCRIT Vashi
}

\begin{abstract}
Predictive analytics helps analyze past events to identify and extract patterns and populations vulnerable to natural calamities. Large number of supervised and unsupervised approaches can be used to identify at risk areas and improve predictions of future events. In addition, predictive analytics techniques can also provide insight for understanding the economic and human impact of natural calamities. Heavy rainfall prediction is a major problem for meteorological department and it is closely associated with the economy and human life. Here the natural disasters we will be focusing on are floods and droughts which are encountered by people across the globe every year. One of the leading causes for this is rainfall. Thus, our main focus will be on rainfall prediction with the help of different parameters and how this prediction can further help us .
\end{abstract}

Keywords: ANN, SLFF, SVM, MLFF, BPNN, Deep learning

\section{INTRODUCTION}

Predictive analytics is the branch of the advanced analytics which is used to make predictions about unknown future events. Predictive analytics uses many techniques from datamining, statistics, modeling, machine learning, and artificial intelligence to analyze current data to make predictions about the future. These predictive analytics can be used for disaster management. The disasters that are focused on here are floods and droughts. The parameter that is considered is rainfall. The meteorological department uses the Climate Forecast System (CFS) model Produced by several dozen scientists under guidance from the National Centers for Environmental Prediction (NCEP), USA. This model uses the global interaction between Earth's oceans, land, and atmosphere.

\section{AIM AND OBJECTIVE}

Fluctuation in rainfall timings and its quantity makes rainfall prediction a challenging task for meteorological scientists. In this project the aim is to analyze and predict the rainfall patterns in India. This prediction done can be used for further application in agriculture to decide the irrigation pattern. Rainfall is caused by a variety of meteorological conditions and the mathematical model for it is non-linear. The objective of the project is to start with the basic data analysis to understand the dataset i.e. to understand the rainfall patterns in India. Using this analysis further step of applying various classification algorithms to understand their accuracy. After considering a case study the deviations in the data is to be found out.

\section{PROPOSED SYSTEM}

Data is collected through various sites available on the Internet. Data preprocessing is done on the data collected. Basic data analysis is done on this final dataset. Data visualization is done for a specialized case. The deviation is checked and the required parameters are to be considered. The proposed system uses different classification algorithms like Neural Networks, Support Vector Machine, regression for prediction. These algorithms are further optimized by adding more parameters. A tool is proposed to be developed to be used for prediction of rainfall.

\section{PREDICTION THROUGH ANN}

ANN is a computational model that is inspired by the human brain. ANN contains a big number of interconnected neurons, which mostly operate in parallel, and are well structured. Categories of neural networks are either single layer or multi-layer. Layer between input layer and output layer is called as hidden layer. A single-layer feed forward (SLFF)neural network consists one input layer whose nodes have weights assigned and one output layer. A multi-layer feed-forward (MLFF) neural network architecture can be developed by adding hidden layers in SLFF neural network. Back-Propagation Neural Network:BPNN is made of MLFF neural network which contains one input layer, hidden layers an done output layer. BPNN architecture with one hidden layer. The ultimate goal of BPNN is to decrease the calculated error obtained from the difference between the calculated output and desired output of the neural network by adjusting the weights after each iteration. So in BPNN, each information is propagated in backward direction until the calculated error is very small or zero. There are three phases of BPNN training:(a)using FFNN for training process of input. Adjustment of weights and nodes are made in this phase, (b) to calculate the error, and (c) modification of weights. ANN model has great ability to learn by doing proper adjustment of these parameters for achieving the desired output. During the training process, this output may fit to the data very well, but it may provide poor results during the testing process. This suggests that the neural network may not generalize well. This might be because of over fitting or over training of data, which can be controlled by analysing the error during training process and stopping the process when the error reaches a minimum threshold with respect to the testing set. Alternate option to make the neural network generalize enough is by doing small changes in the number of layers and neurons in the inputs, without changing the output components. However, best neural network architecture selection is a heuristic approach. Solution is to keep the architecture of neural network relatively simple and small, because complex architectures are much more prone to over fitting. 


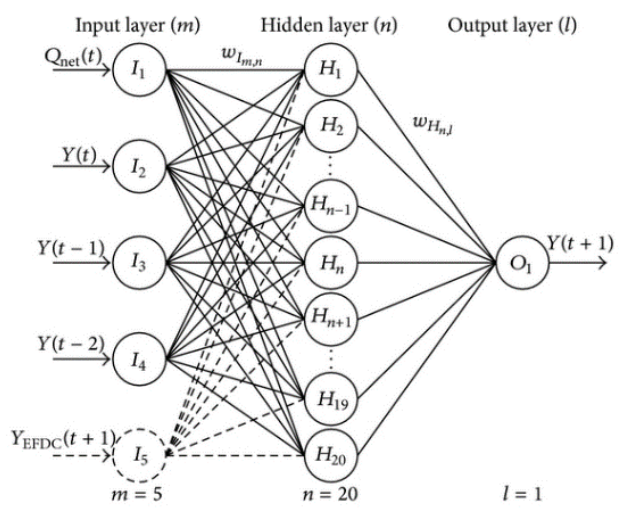

Figure 1. Artificial Neural Network

\section{SUPPORT VECTOR MACHINE (SVM)}

Support Vector Machine is also one type of feed forward network. Support vector machines are applicable for tasks like pattern classification, nonlinear regression etc. Support Vector Machines were created by Vapnik and his coworkers which has been used for supervised learning due to - (i)Better generalization ability than other NN models (ii) SVM solution is identical, optimal and absent from local minima(iii) Applicable to non-vectorial data (Strings and Graphs)and (iv) Very few parameters are needed for tuning the learning machine

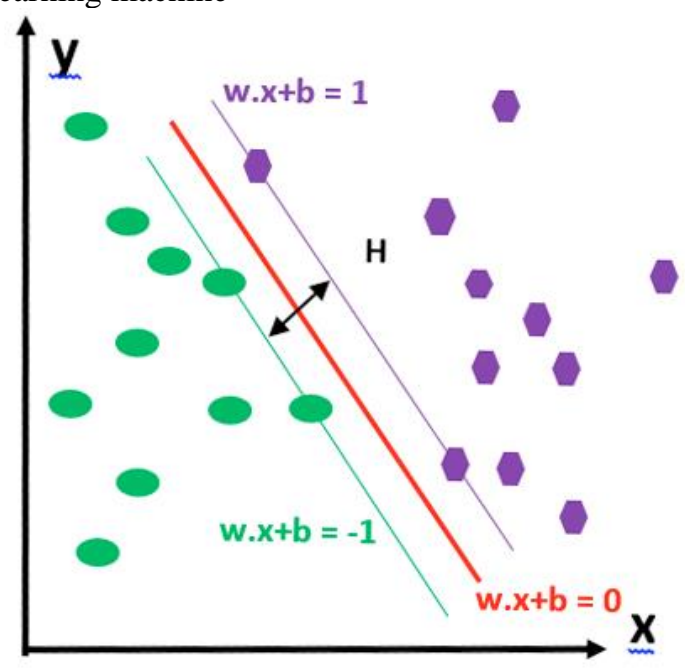

Figure 2. Support Vector Machine

\section{CONCLUSION}

The estimation of rainfall is of great importance in terms of water resources management, human life and their environment. It can be met with the incorrect or incomplete estimation problems because rainfall estimation is affected by the geographical and regional changes and properties. The project considers basic data analysis, the data visualization for a state and a case study. Various classification algorithms are applied. Due to nonlinear relationships in rainfall data and ability of learning from the past makes Artificial Neural Network a preferable approach from all available approaches. Further parameters to be added to get a more accurate prediction of rainfall.

\section{REFERENCES}

[1] P. Goswami and Srividya, "A novel Neural Network design for long range prediction of rainfall pattern," CurrentSci.(Bangalore), vol. 70, no. 6, pp. 447-457, 1996.

[2] C. Venkatesan, S. D. Raskar, S. S. Tambe , B. D.Kulkarni, and R. N. Keshavamurty, "Prediction of all India summer monsoon rainfall using Error-Back-PropagationNeural Networks," Meteorology and Atmospheric Physics,pp. 225-240, 1997.

[3] V. K. Somvanshi, O. P. Pandey, P. K. Agrawal,N.V.Kalanker1, M.Ravi Prakash, and Ramesh Chand,"Modeling and prediction of rainfall using Artificial NeuralNetwork and ARIMA techniques," J. Ind. Geophys. Union,vol. 10, no. 2, pp. 141-151, 2006.

[4] S. Chattopadhyay and M. Chattopadhyay, "A Soft Computing technique in rainfall forecasting," Int. Conf. on IT, HIT, pp. 19-21, 2007

[5] S. Chattopadhyay and G. Chattopadhyay, "Comparative study among different neural net learning algorithms applied to rainfall time series," Meteorological application., vol. 15,no. 2, pp. 273280, 2008. 